\title{
In vivo evaluation of nerve guidance channels of PTMC/PLLA porous biomaterial
}

\author{
Radoslaw A. Wach ${ }^{1}$, Agnieszka Adamus ${ }^{1}$, Karolina Kowalska-Ludwicka² ${ }^{2}$ Bartlomiej Grobelski ${ }^{3}$, \\ Jaroslaw Cala ${ }^{4}$, Janusz M. Rosiak ${ }^{1}$, Zbigniew Pasieka ${ }^{4}$
}

\author{
${ }^{1}$ Institute of Applied Radiation Chemistry, Faculty of Chemistry, Technical University \\ of Lodz, Lodz, Poland \\ 2Institute of Technical Biochemistry, Lodz University of Technology, Lodz, Poland \\ ${ }^{3}$ Faculty of Pharmacy, Medical University of Lodz, Lodz, Poland \\ ${ }^{4}$ Department of Experimental Surgery, Medical University of Lodz, Lodz, Poland
}

Submitted: 3 September 2012

Accepted: 26 December 2012

Arch Med Sci 2015; 11, 1: 210-219

DOI: $10.5114 /$ aoms.2013.34732

Copyright @ 2015 Termedia \& Banach

\section{Abstract}

Introduction: Peripheral nerve disruptions, frequently occurring during limb injuries, give rise to serious complications of patients recovery resulting from limitations in neural tissue regeneration capabilities. To overcome this problem bridging techniques utilizing guidance channels gain their importance. Biodegradable polymeric tubes seem to be more prospective then non-degradable materials - no necessity of implant removal and possibilities of release of incorporated drugs or biologically active agents that may support nerve regeneration process are the main advantages.

Material and methods: Polymer blend of commercial poly(L-lactic acid) (PLLA) and in-house synthesized poly(trimethylene carbonate) (PTMC) were processed in an organic solvent - phase inversion process on a supporting rod - to form a guidance porous tube of $1.1 \mathrm{~mm}$ inner diameter. In vivo experiments on rat's cut femoral nerve by using either the tubes or end-to-end suturing (control group) involved 22 and 19 rats, respectively. Motor recovery of operated limbs, neuroma occurrence and histopathology of explanted nerves were evaluated after 30, 60 and 90 days of implantation.

Results: Motor recovery of the limbs was of similar rate for the two animal groups. The neuroma formation was evident in over $90 \%$ control specimens, while for the bridging group it was less than $40 \%$ of all evaluable samples $(p=0.0022)$. Biocompatibility of applied materials was affirmed by moderate tissue response.

Conclusions: Application of the biodegradable PLLA/PTMC polymeric tubes effectively supports regeneration of discontinued nerves. The applied material prevents neuroma formation, by reducing the scar tissue formation time and, thus, accelerating the process of neural tissue restoration.

Key words: peripheral nerve regeneration, poly(L-lactic acid), poly(trimethylene carbonate), tubulisation.

\section{Introduction}

Peripheral nerve lesion is one of the most important and frequent types of injuries associated with accidents. Severe lesions of the peripheral nerves may result in incomplete axonal regeneration and permanent disability in patients' limbs. Total recovery of nerve function after repair is rarely achieved in spite of the intrinsic capacity of nerve fibres to regenerate after axotomy [1-3]. The repair of peripheral nerve lesions has been attempted in a few different ways, which all have in common the goal of

\author{
Corresponding author: \\ Radoslaw A. Wach MD, PhD \\ Institute of Applied \\ Radiation Chemistry \\ Faculty of Chemistry \\ Technical University of Lodz \\ 15 Wroblewskiego St \\ 90-924 Lodz, Poland \\ Phone: +48 426313164 \\ E-mail: wach@mitr.p.lodz.pl
}


directing the regenerating nerve fibres into the proper distal endoneurial tubes [4]. The strategies developed for nerve repair can be roughly classified into two categories. One is end-to-end suturing of the nerve stumps, which includes direct coaptation of two stumps and end-to-side (ETS) repair. Bridging is the other technique, which includes grafting and tubulisation [5].

Direct nerve repair, such as epineural or fascicular suturing, is applied when there is no gap at the lesion site [6]. For complete lesions, primary endto-end repair is preferred when possible and has the best prognosis [7]. In cases of long segmental defects, where only the distal stump is available and autologous nerve grafts are not available or have not worked, ETS repair is an alternative. The basic concept of ETS nerve repair is to obtain nerve fibre regeneration to the distal stump of a transected nerve by inducing collateral axonal sprouting from a healthy neighbour (donor) nerve. However, ETS nerve repair results in intraneural scarring and leads to a significantly lower number of myelinated fibres and smaller fibre diameters compared with end-to-end repair [8].

In cases of severe injuries accompanied by long defects in the peripheral nervous system, a grafting technique is often applied. Autogenous graft is one of the common clinical procedures used to connect the proximal and distal section of the nerve. The success of autologous nerve grafts is due to the presence of Schwann cells and basal lamina endoneurial tubes, which provide neurotrophic factors, and endoneurial tube surface adhesion molecules to regenerating axons. Disadvantages of this technique include donor site morbidity, e.g. scarring and, occasionally, neuroma pain, and the need for multiple operations.

The main drawback of this method, however, is the fact that the patient suffers additional trauma related to the surgery of the nerve donor site, and afterwards is lacking functionality and filling in the harvested tissue.

In addition, some biological constraints cannot be overcome by the progress of microsurgery. For instance, endoneurial tubes cannot be exactly approximated, leading to mismatching of regenerating axons and consequent nonspecific incomplete reinnervation and poor recovery of nerve function. The subsequent overgrowth of connective tissue that forms painful nodules (neuromas) at the site of concretion most often constitutes a risk of cancerogenesis and thus forces the surgeons to conduct further corrective operations. Having the tools to regenerate the axons faster and in the proper manner, one could avoid such complications [9-11]. Nerve autografting is the most successful method for nerve repair. However, bio-engineered grafts have become a promising alternative, as they can incorporate all the new developing strategies for nerve regeneration, which continually develop side by side with the knowledge of the mechanism of regeneration [12].

A wide variety of materials have been suggested for the production of artificial devices for nerve repair, including biocompatible non-degradable and degradable polymeric materials [4]. Possible disadvantages of utilization of artificial conduits include introduction of foreign material into the body, which may cause a strong immune system response, but only in the case when the material is of unproved biocompatibility or the products of its degradation are harmful to the organism. Traumatization during the surgery may be avoided or at least limited by microsurgical techniques and highly skilled neurosurgeons. The prospects for the method are very promising, overwhelming possible disadvantages [13]. On the other hand, the benefits of utilization of biodegradable synthetic polymers, besides the aforementioned avoidance of multiple surgery, are mainly due to tunable physical and chemical properties such as mechanical strength and porosity that may influence degradation behaviour [4].

Poly(L-lactic acid) (PLLA) is an example of an aliphatic polyester and high-strength and high-modulus polymeric biomaterial of acceptable biocompatibility and inherent biodegradability with several biomedical applications. The polymer degrades in an aqueous environment through hydrolysis of ester bonds in the main chain, and the ultimate degradation product, of mainly lactide acid, is metabolized by the organism into carbon dioxide and water $[14,15]$. To introduce modifications to rigid PLLA, poly(trimethylene carbonate) (PTMC), a rubbery and flexible material, can be used as a copolymer of PLLA or a co-component in a blend [16-19]. The PTMC is a linear, amorphous polymer with a glass transition temperature of approximately $-15^{\circ} \mathrm{C}$. It has been investigated for potential biomedical applications in soft tissue engineering and in drug delivery systems. The polymer is relatively stable towards hydrolysis, although it degrades in vivo by an enzymatic surface erosion mechanism [20].

The main objective of the present study was to analyse in vivo performance of guidance channels made of PTMC/PLLA blended polymers applied for the reconstruction of damaged peripheral nerves. Development of manufacturing technology of the tubes and their physical-chemical examination were described earlier [21]. Appropriate mechanical properties and lack of cytotoxicity evaluated through screening biocompatibility testing confirmed suitability of the chosen biomaterial for in vivo evaluation towards the proposed application. Utilization of neurotubes is expected to limit the formation of traumatic neuromas, clinically observed as small nodules at the anastomosis site, which are often 
Table I. Molecular weight and thermal properties of PLLA and PTMC polymers used in the experiments

\begin{tabular}{|lcccccc|}
\hline Polymer & $\mathrm{Mn}^{1}[\mathrm{kDa}]$ & $\mathrm{Mw}^{1}[\mathrm{kDa}]$ & $\mathrm{PD}$ & $\mathrm{Tg}^{2}\left[{ }^{\circ} \mathrm{C}\right]$ & $\mathrm{Tm}^{2}\left[{ }^{\circ} \mathrm{C}\right]$ & $\mathrm{DC}^{2}$ \\
\hline PLLA & 79 & 155 & 1.9 & 60.2 & 150.6 & 30.9 \\
\hline PTMC & 63 & 94 & 1.5 & -18.6 & - & \\
\hline
\end{tabular}

$M n$ - number and $M w$ - weight average molecular weights, $P D$ - polydispersity index, $T g$ - glass and $T_{m}$ - melting transition temperatures, $D C$ degree of crystallinity [19]. ${ }^{1}$ Determined by gel permeation chromatography (GPC); ${ }^{2}$ determined by differential scanning calorimetry (DSC).

formed due to an insufficient reparative process [22]. Development of neuromas significantly lowers the ability of neural conductivity restoration, making full functional recovery of disabled nerves much longer or even impossible. Therefore, implanted prostheses should accelerate the process of regeneration compared to the standard surgical method of end-to-end connection.

\section{Material and methods}

\section{Materials}

The PTMC is not available commercially, so the polymer was synthesized in our laboratory. The method for PTMC polymerization was based on that described in the literature for similar compounds such as PLA and PCL [23] and tailored for polymerization of the polycarbonate [21]. In brief, polymerization of trimethylene carbonate monomer (Boehringer Ingelheim, Germany) was carried out in tetrahydrofuran with diethylaluminum ethoxide (Et ${ }_{2} \mathrm{AlOEt}$ ) (Aldrich, USA) initiator under argon atmosphere. Diethylaluminum ethoxide was added to the monomer in the monomer to initiator molar ratio of 1000 and the polymerization was carried out for about $15 \mathrm{~h}$ at room temperature. The obtained polymer was purified by precipitation from chloroform, extensively washed with methanol and dried under reduced pressure at room temperature.

Commercially available poly(L-lactic acid) (PLLA, Dow-Cargill) granules were dissolved in chloroform and purified by precipitation onto methanol and washed with this alcohol. Basic characteristics of utilized PTMC and PLLA polymers are presented in Table I. Other solvents and reagents were of analytical grade, and unless otherwise stated were pur-

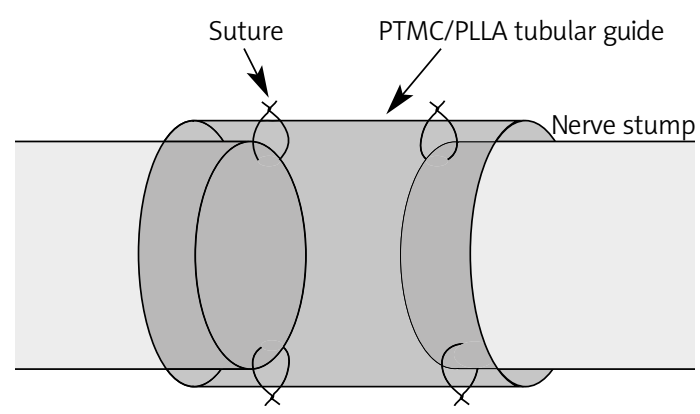

Figure 1. Schematic drawing of the stabilisation of a lesion with PLLA/PTMC nerve guidance channel chased from Sigma-Aldrich and used without further purification.

\section{Tube manufacturing}

The method of nerve regeneration channel fabrication was developed earlier [21]. Polymers of PLLA and PTMC in a $1: 1$ wt. ratio were dissolved in dioxane at a total concentration of 20 wt./vol.\%, resulting in a very viscous liquid. Standard injection needles of $1.1 \mathrm{~mm}$ diameter were repeatedly dipped in polymer solution and in a non-solvent solution of isopropanol and water in $3: 7 \mathrm{vol}$. proportion. Polymer recovery was triggered by phase separation in the non-solvent bath resulting in tubular porous structures of about $30 \mathrm{~mm}$ in length. Polymer tubes were removed from needles, washed extensively in water and alcohol, dried under vacuum and packed separately. The tubes designed for implantation were of $10 \mathrm{~mm}$ or more in length and of $1.1 \mathrm{~mm}$ lumen diameter.

Sterilisation by ionizing radiation with a standard sterilisation dose of $25 \mathrm{kGy}$ was applied. Polymer tubes in vacuum-sealed bags were irradiated by an electron beam from the linear accelerator with a dose rate of about $5 \mathrm{kGy} / \mathrm{min}$.

\section{Surgical procedures}

In vivo analyses were performed on Wistar rats, female, weighing up to $150 \mathrm{~g}$ at the commencement of the experiments. All animals received humane care in compliance with the European Convention on Animal Care, and all the procedures were carried out according to agreement of the Local Ethical Committee of the Medical University of Lodz. Animals were housed one in each cage with free access to food and drinking water.

The stabilisation procedure for control and tubulisation, i.e. 'bridging' groups was applied earlier [24] and is briefly described as follows. In order to proceed with implantation, animals were anesthetised with ketamine (Ketanest $50^{\circledR}$ ) delivered intraperitoneally in a dose of $20 \mathrm{mg} / \mathrm{kg}$ c.c. Sutures applied included Premilene ${ }^{\circledR} 7 / 0$ (BRAUN) and Monosof ${ }^{\mathrm{TM}}$ 4/0 (Syneture) for nerve and skin suturing, respectively.

In the bridging group, comprising 22 rats, the tubes were threaded onto cut femoral nerve, with over $5 \mathrm{~mm}$ space left between the stumps, and stabilised with 4 sutures to the epineurium (Figures 1 and 2 D). In the control group of 19 rats, traditional end-to-end 
connection with single loop suture was applied - Figure $2 \mathrm{~A}$. Observation time was 30, 60 and 90 days. The evaluation of recovery changes was based on postoperative observation of disabled limb movement. To avoid self-injury by eating digits of operated legs (auto-cannibalism), the animals were given analgesic solution (Metamizolum natricum inj., Polpharma S.A.) in doses of $0.5 \mathrm{~g}$ per $100 \mathrm{ml}$ of drinking water every day for 2 months following transection. Subsequently, before explantation at each observation time point the rats were humanely euthanized by administering an overdose of ketamine.

\section{Motor recovery assessment}

The recovery of operated limbs was evaluated on the basis of the Medical Research Council Sys- tem (MRCS) for grading nerve recovery $[25,26]$. The scale based on this system served to estimate the degree of disabled leg movement after 30, 60 and 90 days. The animals were given scores from 1 (low) to 5 (high) at step 0.5 , in order to find the average value describing each investigated group. To avoid subjectivism, the examination and limb movement evaluation were performed by two independent observers.

\section{Histology}

Microscopic evaluation was based on haematoxylin-eosin (HE) staining for tissue-biomaterial interaction as well as S-100 immunohistochemical treatment for the purpose of neuron growth observation. Explanted specimens, i.e., end-to-end con-
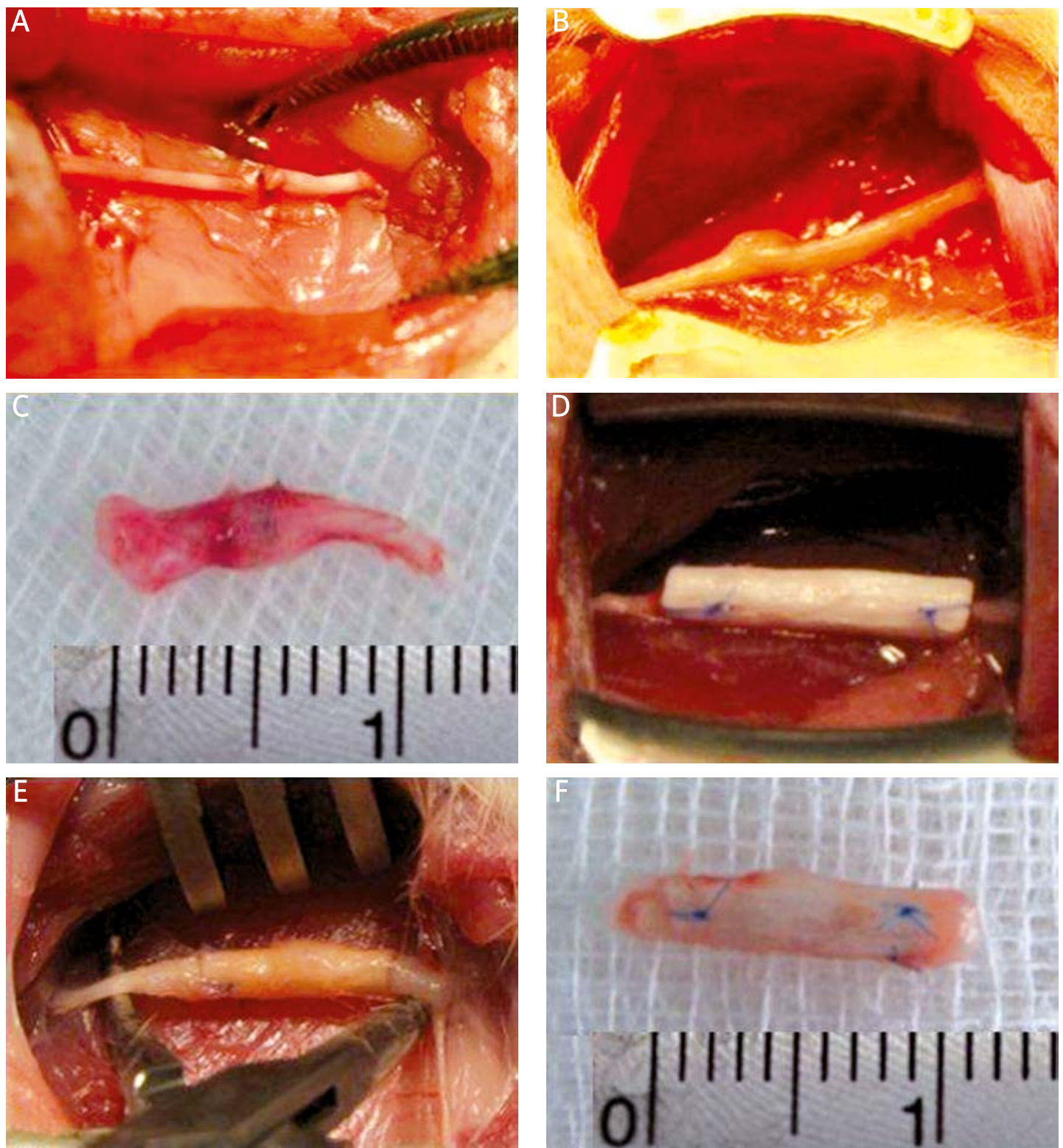

Figure 2. Macroscopic view of end-to-end nerve connection (A-C) and polymeric guidance channels (D-F) operation field and explants: A, D - implantation; B, E - explantation after 30 days; C, F explanted nerves 
nected nerves and the nerves enclosed in the tubes, shown in Figures $2 \mathrm{C}$ and $\mathrm{F}$ respectively, were formalin fixed and then paraffin embedded. Serial $5 \mu \mathrm{m}$ thick tissue sections cut from paraffin blocks were mounted individually onto charged glass slides. One slide of each case was haematoxylin-eosin stained for the purpose of microscopic evaluation of tissue immune response.

Immunohistochemical reaction for S-100 protein was performed in order to evaluate neuron outgrowth and neuroma formation. A positive reaction was considered as simultaneous tint of nucleus and cytoplasm of neural cells (background and fibroblast were negative, only counterstained with haematoxylin). Neuroma formation was described as excessive growth of connective tissue around and within the scar formation site together with the loss of regular structure of nerve fibres (general disorder of growing nerve cells).

\section{Statistical analysis}

For motor recovery results analysis the MannWhitney $U$ test was applied. In the case of neuroma formation examination (S-100 analysis), the low number of evaluable samples in the investigated observation times, made the analysis of changes in neuromas time distribution meaningless. Therefore, the statistical analysis in this point was limited only to the total number of neuromas in each group and applied $\chi^{2}$ test.

\section{Results}

\section{Animal behaviour assessment}

A total of 39 rats underwent surgical treatment. 17 explants were successfully obtained in each group (control and 'bridging') and 12 samples of each group were histologically evaluable, i.e. did not

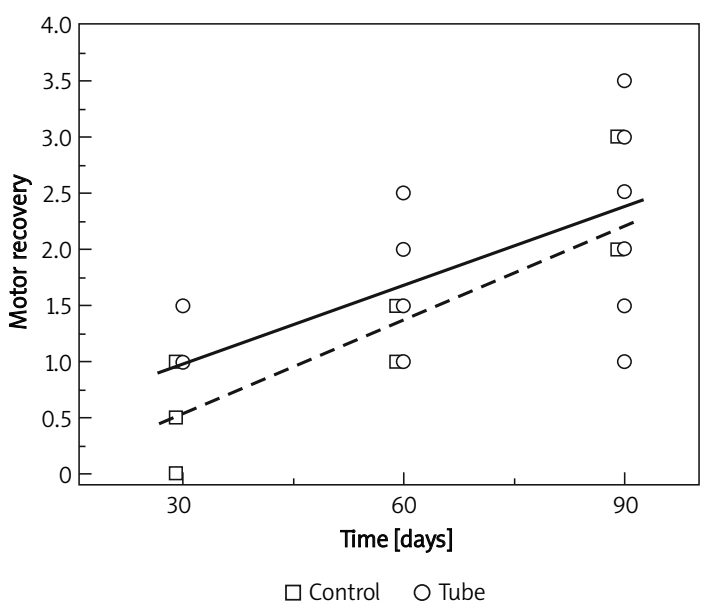

Figure 3. Motor recovery of operated limbs according to MRCS scale for individual animals in control and bridging group (repeated scores are depicted as a single point) undergo any mechanical or procedural damage during preparation, and the image observed under the microscope showed the concretion site. Two rats from the control group died postoperatively due to infection and 3 rats from the bridging group had to be killed because of auto-mutilation. None of the control rats revealed self-injury, while some of the bridging group bit their own digits. In most cases administration of proper dosage of analgesic solution prevented such behaviour.

Motor recovery assessment was performed every 30 days following the surgery up to explantation time. The movement recovery increased relatively slowly in time in both investigated groups (Figure 3). The contracture of disabled digits could be seen in the majority of cases from both groups regardless of the given score. Statistical analysis (Mann-Whitney $U$ test) showed that there was no significant difference $(p>0.05)$ in average leg movement ability between control and polymeric tube groups at each time point.

\section{Explantation}

Considering histological explant analyses of the control group and the bridging group, the samples were evaluated in a total of 17 for each group, that is 2, 5 and 10 specimens after 30, 60 and 90 days respectively.

Explantation of the nerves after 30 days is presented in Figures $2 \mathrm{~B}$ and $\mathrm{E}$, and the exemplary specimens subjected to further evaluation in Figures $2 \mathrm{C}$ and $\mathrm{F}$. The picture of end-to-end connection explant shows the example of a large neuroma that arose in the suturing location, i.e. an irregular bulge was observed at the scar formation site. In the bridging group, explanted PTMC/PLLA tubes did not undergo any physical changes, retaining their original size and shape. They seemed to be softer or more flexible, and they were well integrated with the surrounding tissue, and eventually overgrown by connective tissue with newly formed small blood vessels. At the end of each observation period, i.e. during explantation and removal of the samples, no visible signs of either acute inflammation or infection were detected.

\section{Histological assessment}

Analysis of the immune response to the implanted biomaterial was conducted by light microscope observations of the HE stained specimens taken from PTMC/PLLA explanted tubes. Three areas were considered, as indicated in Figure 4: (a) the outside surface of the tube, (b) within the wall of the porous tube and (c) between the tube and the regenerating nerve. The extent of tissue response was divided into three levels: weak (average granulation tissue thickness - 1-2 cells), moderate (aver- 


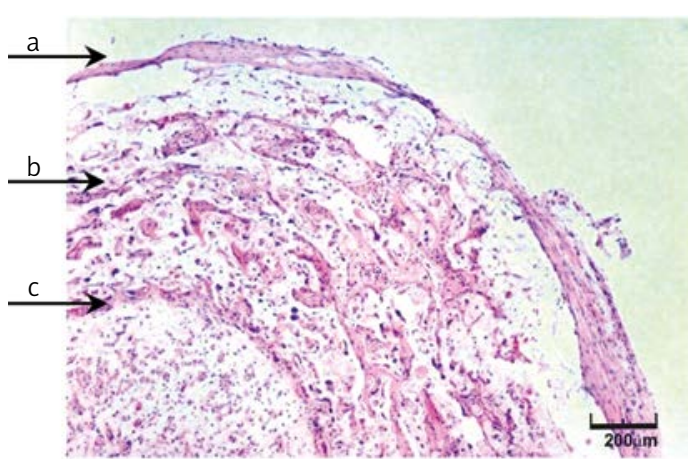

Figure 4. Tissue response evaluation in the HEstained cross-section of the PTMC/PLLA sample explanted after 90 days: (a) on the outside surface of the tube - weak, (b) within the wall of the porous tube - moderate, and (c) between the tube and the regenerating nerve - moderate (magnification 60x)

age granulation tissue thickness $-3-4$ cells) and intensive (thickness greater than 4 cells).

The micrographs revealed that the severity of the granulation on the outer surface of the implants decreased with time of observation from moderate after 30 days to weak at the $90^{\text {th }}$ day of observation. Granulation within the walls of the neural guides was observed in the case of all time groups (example in Figure $5 \mathrm{~A}$ ). Lymphoid cell invasion into the walls progressed with time of observation. The severity of the inflammatory response increased from weak after 30 days to moderate at the $90^{\text {th }}$ day. There was also a moderate to weak (depending on the samples) intensity of tissue reaction between the tube and the nerve that remained unchanged for the whole period of observation.

S-100 staining analysis was performed for the evaluable samples (i.e. not damaged during the analytical procedure) in order to examine the extent and quality of concretion. A dark brown stain in the microscopic image (Figures 5 B and C) indicates the nerve fibres, whereas pale beige is an indication of connective tissue. Analysing the composition of the explanted tissue fragments, one could define whether the regenerative process proceeded correctly and the fibres grew properly or there were some disturbances, such as infiltration with scar tissue, excessive inflammation or lack of concretion, and the formation of neuroma. Since instant suturing of the stumps was applied in the control group, the connection was achieved already at the operation time. Nevertheless, during the course of recovery, frequent occurrence of neuroma, as depictured in Figure $5 \mathrm{~B}$, was detected in the control group. In contrast, $5 \mathrm{~mm}$ or larger gap between the two ends of cut nerve remained after surgery in the bridging group. The restoration of damaged nerves inside the tubes occurred already in the first observation period, i.e. within 30 days after implantation. No further changes of the condition of restored
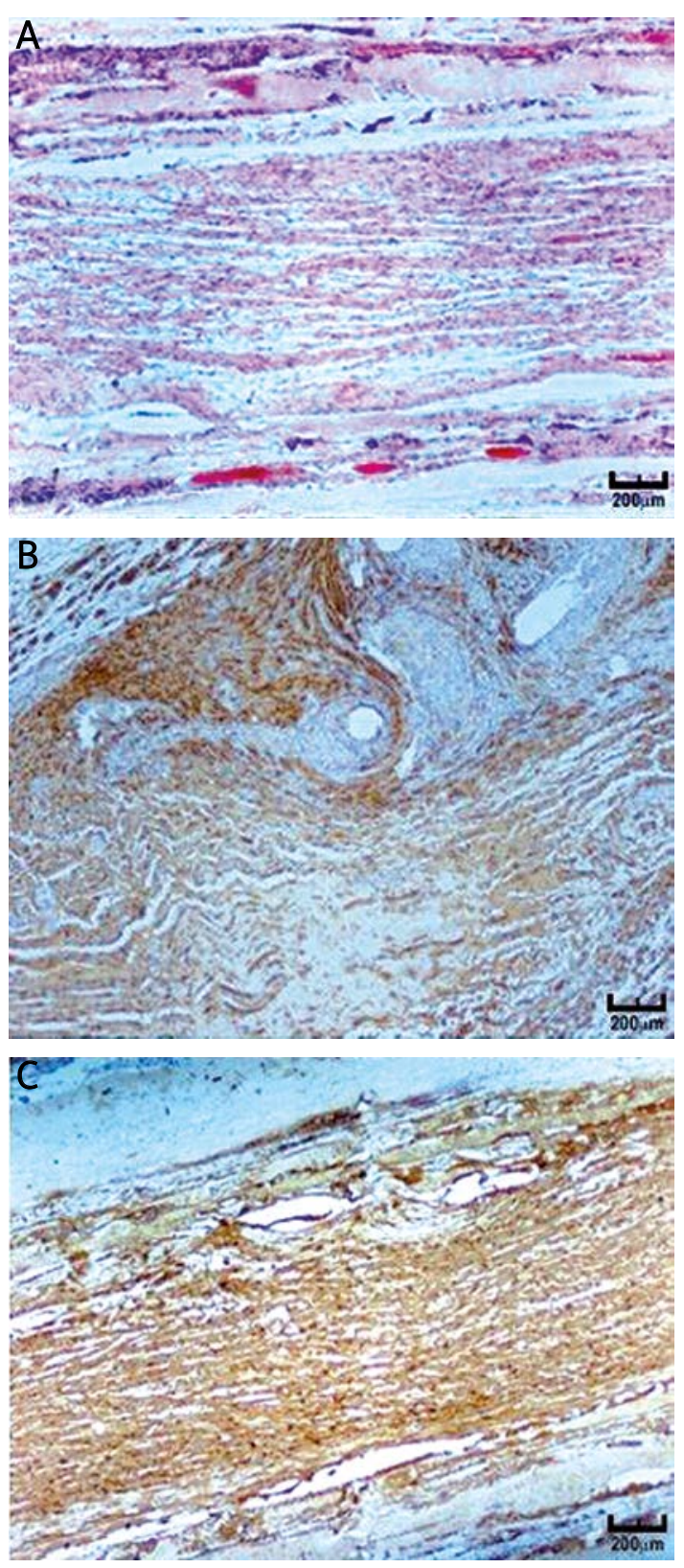

Figure 5. PTMC/PLLA tubes $A, C$ and control $B$ explanted after 90 days. A - Low tissue reaction, HE staining; B - neuroma in control specimen, S-100 staining; $\mathbf{C}$ - properly regenerated nerve without neuroma inside the tube, S-100 staining (magnification $60 x$ )

nerves, as indicated with S-100 staining, were observed in 60 and 90 days.

A notable difference in neuroma occurrence between experimental groups was observed. The tubes made of PTMC/PLLA blend applied in vivo considerably lowered the formation of neuroma as compared to the control group. The overgrowth of connective tissue and disorganisation of neural structures was evident in over $90 \%$ of control specimens, while for the bridging group it was less than $40 \%$ of all evaluable samples. It is explicable that the 


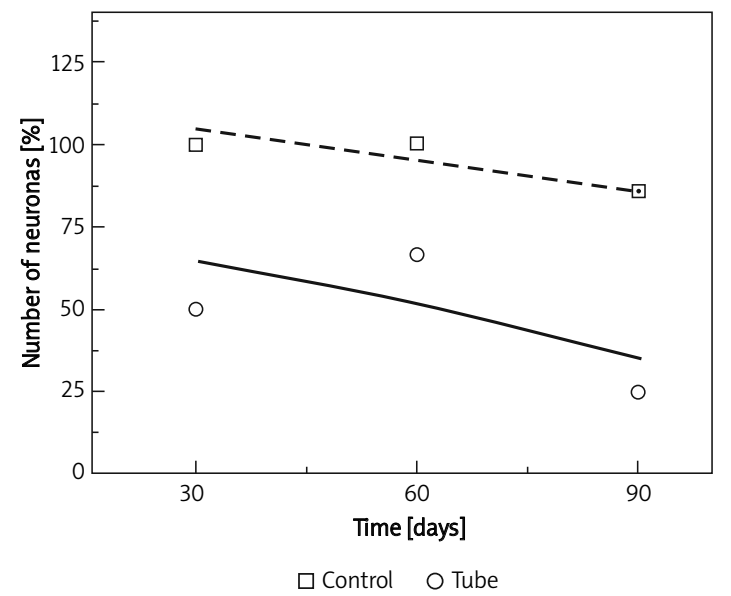

Figure 6. Changes in the frequency of neuroma occurrence for the groups of PLLA/PTMC tube material and control

number of formed neuromas decreased with respect to time, as the regeneration proceeded (Figure 6).

Statistical analysis was confined to the total number of neuromas occurring in each group and $\chi^{2}$ test was applied. The results showed that the difference in the total number of neuromas between investigated groups was statistically significant $(p=0.0022)$.

\section{Discussion}

\section{Nerve guidance tubes - polymers and sterilisation considerations}

Guidance channels of PTMC/PLLA blends were produced without utilisation of sophisticated equipment, yet the resulting implants combined considerable mechanical strength, mainly due to the PLLA, and high flexibility because of the incorporated PTMC as well as the presence of porosity in the tube wall [21]. These two polymers of established biocompatibility have already been investigated for utilisation as nerve guidance tubes. The tubes were produced from neat PTMC of high molecular weight by salt leaching and fibre winding techniques but they were not evaluated in vivo [27]. An immersion coating method followed by porogen particle leaching was also applied to copolymers of PTMC-COPLLA in order to obtain porous tubes, which resembles the present investigations [28]. Nevertheless, those channels have not been tested in vivo for their intended application of nerve regeneration assistance; thus it was unknown whether the PTMC/PLLA polymers would support the regeneration of discontinued nerve. Therefore, the current approach has a solid background in the literature and application of the PTMC/PLLA material in animal studies is justified.

Besides perfect biocompatibility, a crucial factor resulting in successful application of the bio- material in vivo is microbiological cleanliness, which guarantees safety of the implant. Sterilisation by radiation technique was chosen as the most appropriate method for the polymer composition employed for fabrication of the guides supporting nerve reconstruction. This was due to the fact that PTMC and PLLA are characterized by low glass transition temperatures of about $-18^{\circ} \mathrm{C}$ and $60^{\circ} \mathrm{C}$ respectively, and softening temperature - PTMC becomes very soft and tacky over $50^{\circ} \mathrm{C}$. Additionally, polylactide undergoes hydrolysis in a wet environment, especially at an elevated temperature. Therefore the tube would deform during steam autoclaving, and some reduction of PLLA molecular weight was expected due to rapid hydrolysis of ester bonds. Moreover, the morphology of the tube walls is porous, and therefore such a popular low temperature method of biodegradable synthetic polymer sterilisation as ethylene oxide was discarded here, since the sterilizing gas might not be able to reach the entire porous structure of the implant or diffuse out after the process. Studies on the influence of irradiation on these polymers, used here as a sterilisation tool for the neurotube implants, were described previously [21]. It is known that PTMC processed separately and in the blend tends to increase in molecular weight during irradiation by ionizing radiation [29]. Oppositely, polylactides degrade readily, so the important factor is their initial molecular weight that would determine the end product mechanical properties after radiation sterilisation [30]. The results of previous investigations on PTMC/PLLA blends allowed us to choose the optimal compositions of a material that is biodegradable and biocompatible and whose functioning does not undergo detrimental changes after sterilisation with electron beam (EB) irradiation [21, 31]. It was concluded that physical chemical parameters of the tubes after EB sterilisation were sufficient, and based on the positive results of in vitro biocompatibility evaluation animal studies were undertaken.

\section{Bridging the lesion}

Critical stages when the tubes are required to maintain their mechanical strength are the implantation procedure, i.e., suturing, and the initial postoperational period. After a short time following the operation, besides some movement of the limb, there is no considerable tension induced by nerve stumps sutured to the tube. Therefore, some changes in mechanical properties of the tubes, which occur due to biodegradation of the comprising polymers, are acceptable [31]. During the course of degradation - principally hydrolytic for PLLA and enzyme-assisted for PTMC - the tensile strength and the strain would reduce gradually; nevertheless, the guide continuously provides space for 
regeneration for at least several months. In the present investigations the maximum period of 90 post-operative days was chosen. This was sufficient for indicating a potential response of the surrounding tissue, e.g. signs of inflammation or infection, or probable occurrence of neuromas, and allowed for complete nerve regeneration inside the channel. Since no rejections of implants were recorded, porous PLLA/PTMC material is concluded to be well accepted by a host organism and suits the requirements of successful treatment of neural disorders.

Autocannibalism, the main complication that occurred during the undertaken trials, probably indicated accumulation of neurotrophic factors. This was observed in the bridging group, but not in the control one. Following the literature, this fact may suggest that PLLA/PTMC tubes support the accumulation of growth factors promoting the regeneration of a damaged nerve [32, 33]. This inference is of great importance especially for porous materials. Taking advantage of the fact that the applied implant will be degraded, one may fit the time of this degradation to the time of regenerating tissue demand for the specific neurotrophic factors. Having the opportunity to immobilize selected neurotrophins within the polymer, which is going to be the forthcoming phase of the experiments, it is possible to release those factors at a predetermined time of the restoration process, so as to stimulate the regeneration more efficiently.

\section{Motor recovery}

The main factor responsible for the less evident difference in motor function recovery between the control and the bridging groups was probably the invasiveness of the surgical procedure and prolonged duration of the surgery, since tubulisation was much more time consuming than traditional suturing. As a consequence, muscle damage and subsequently their partial atrophy might have occurred. However, in order to confirm such observations sham-operated rats should be included in the investigative process. In the present investigations, due to taking special care to reduce the number of animals, including an additional control group was not accepted.

The contracture of disabled digits could be seen in the majority of cases from both groups regardless of the given score. In this type of injury, prolonged intense rehabilitation is a crucial factor for recovery of motor functions of the disabled limb. Present tests did not cover rehabilitation of injured limbs of the animals, but we expect that application of a rehabilitation procedure might greatly improve recuperation from the surgical intervention.

\section{Histology}

Response of the surrounding tissue in the bridging group was acceptable, not exceeding a moderate level regardless of the observation time. Since there is no possibility to avoid infiltration with body fluids, the growth of connective tissue inside the tube was expected. However, if the stabilization method and the scaffold itself are conducive for the growth of axons, the growing cone pushes the obstructing fibres of connective tissue aside. Despite the connective tissue infiltration in the early stages after the surgery, the regeneration proceeded effectively, the axons were properly guided, and the clusters that had formed between the stumps after some time were visible only as tissue surrounding the nerve. For that reason, histologically only the concretion by means of connective tissue, not the nerve surrounded by these fibres, was considered to be a neuroma. Both macroscopic and histological observation confirmed that bridging by means of applied biodegradable tubes prevented the excessive proliferation of connective tissue and provided accurate guiding of the growing axons. Nerves regenerated inside the tubes appeared to be regular bundles of axons identical to a healthy nerve. The fibres regained their regular structures by fusing during the first 30 days of wound healing. However, looking at differences between time groups, the process of total nerve regeneration, in this case, seems to take more than 60 days. The immunohistochemical outcome confirms that connective tissue penetrating the concretion site within the tube infiltrates it intensively within 60 days after the surgery, and is further gradually replaced by the growing number of properly regenerated axons, becoming the basis of accurate final restoration of the tissue. Neuroma formation tends to occur in the control group as the result of inadequately slow regeneration together with mismatch in the position and size of nerve (axon) bundles. Subsequent muscle atrophy of the distal part of the limb makes functional recovery very difficult or even impossible. Based on this knowledge it was rational to undertake actions in order to develop alternative methods of nerve lesion bridging, i.e. PTMC/PLLA tubulisation.

There were no visible signs of material-induced infection. This might have occurred if the applied material was of questionable biocompatibility and/or sterility or if the rate of polymer biodegradation had been improperly selected, thus leading to accumulation of acidic degradation products [34]. Some inflammatory response of the tissue is due to the fact that the applied polymer is highly porous, enabling the gradual penetration of its structure by host tissue and thus provoking the delayed immune response within the walls of the 
tubes. Porous scaffolds are usually susceptible to host tissue overgrowth, and the present material of PTMC/PLLA gives some moderate granulation tissue formation at the implant site. Such granulation should not last longer than the degradation time, and should be diminished in the course of replacing the degrading polymer with the host connective tissue.

It is known that the material applied to build the tubes, comprising two polymers, degrades even in vitro by losing its tensile strength in less than 5 months, followed by mass loss, and total degradation occurred in less than a 1-year period [35]. In the in vivo environment, two distinct processes drive biodegradation of the two different polymeric components. Simple hydrolytic degradation takes place for the PLLA, when random cleavage of ester bonds causes steady reduction in polymer molecular weight in bulk. On the other hand, the oxidation by reactive oxygen compounds secreted by macrophages erodes the PTMC surface. [20]. As a result of the utilized composition, i.e. 50/50 PTMC/PLLA, even for a porous structure, no apparent indication of degradation was observed in $\mathrm{HE}$-stained specimens within 90 days of implantation. Nevertheless, we assume that the polymer biodegradation products released inside the tube, which are difficult to quickly transport outside, do not affect the inherent nerve regeneration. It is expected that the surrounding tissue response will decrease in the due course of tissue regeneration. The material offers the option to stimulate the regeneration and wound healing more effectively by manipulating the time of degradation, that is altering the ratio and molecular weight of polymers. Taking advantage of this possibility, it is expected that the application of stimulating factors immobilized within the tubes' porous walls will hold even more potential.

In conclusion, nerve guidance tubes produced from polymers of PTMC/PLLA blend were applied in an approximately $5 \mathrm{~mm}$ wide lesion of rat femoral nerve. The results of nerve recovery assisted by this bridging technique were compared to classical endto-end connection at 30,60 and 90 days observation time. The polymeric tubes were produced by a straightforward and effective method of phase separation but their functionality in vivo appeared to be very satisfactory. Besides well-known advantages of tubulisation over transplantation, we should stress the importance of tube strength and flexibility, which were adjusted for this particular application by selecting the proper composition of durable, i.e. PLLA, and flexible, i.e. PTMC, polymers, and wall porosity allowing for better fluid transportation and ingrowth of the surrounding tissue and its integration. The relatively low level of tissue response confirmed good biocompatibility of the applied biomaterial. Application of the tubes effectively prevented the formation of neuroma, therefore enhancing the process of nervous tissue reconstruction and reducing scar tissue formation. The tubes are also considered to be conducive to accumulation of neurotrophic factors inside, which contributes to faster recovery of the nerves.

\section{Acknowledgments}

This work has been partially financed by the European Commission project 'NerveRegeneration' MIGR-CT-2007-206269 within 7FP and by the Ministry of Science and Higher Education, Poland: 1953/B/P01/2008/35 and SPB grant 843/7 PR UE/2008/7.

\section{Conflict of interest}

The authors declare no conflict of interest.

\section{References}

1. Evans GRD. Peripheral nerve injury: a review and approach to tissue engineered constructs. Anat Rec 2001; 263: 396-404.

2. Geuna S, Raimondo S, Ronchi G, et al. Histology of the peripheral nerve and changes occurring during nerve regeneration. Int Rev Neurobiol 2009; 87: 27-46.

3. Deumens R, Bozkurt A, Meek MF, et al. Repairing injured peripheral nerves: bridging the gap. Prog Neurobiol 2010; 92: 245-76.

4. Ciardelli G, Chiono V. Materials for peripheral nerve regeneration. Macromol Biosci 2006; 6: 13-26.

5. Dahlin LB. Techniques of peripheral nerve repair. Scan J Surg 2008; 97: 310-6.

6. Wolford LM, Stevao EL. Considerations in nerve repair. Proc (Bayl Univ Med Cent) 2003; 16: 152-6.

7. Diao $E$, Vannuyen T. Techniques for primary nerve repair. Hand Clin 2000; 16: 53-66.

8. Yuksel F, Karacaoglu E, Guler MM. Nerve regeneration through side-to-side neurorrhaphy sites in a rat model: a new concept in peripheral nerve surgery. Plast Reconstr Surg 1999; 104: 2092-9.

9. Doolabh VB, Hertl MC, Mackinnon SE. The role of conduits in nerve repair: a review. Rev Neurosci 1996; 7: 47-84.

10. Ichihara S, Inada Y, Nakamura T. Artificial nerve tubes and their application for repair of peripheral nerve injury: an update of current concepts. Injury 2008; 39: 29-39.

11. Sun H, Yang T, Li Q, et al. Dexamethasone and vitamin B12 synergistically promote peripheral nerve regeneration in rats by upregulating the expression of brain-derived neurotrophic factor. Arch Med Sci 2012; 8: 924-30.

12. Liu GB, Cheng YH, Feng YK, et al. Adipose-derived stem cells promote peripheral nerve repair. Arch Med Sci 2011; 7: 592-6.

13. Jiang X, Lim SH, Mao HQ, Chew SY. Current applications and future perspectives of artificial nerve conduits. Exp Neurol 2010; 223: 86-101.

14. Engelberg I, Kohn J. Physico-mechanical properties of degradable polymers used in medical applications: a comparative study. Biomaterials 1991; 12: 292-304.

15. Amecke B, Bendix D, Entenmann G. Resorbable polyesters: composition properties applications. Clin Mater 1992; 10: 47-50.

16. Pego AA, Pot AA, Grijpma DW, Feijen J. Physical properties of high molecular weight 1,3-trimethylene carbonate and 
D,L-lactide copolymers. J Mater Sci Mater Med 2003; 14: 767-73.

17. Zhang Z, Grijpma DW, Feijen J. Triblock copolymers based on 1,3-trimethylene carbonate and lactide as biodegradable thermoplastic elastomers. Macromol Chem Phys 2004; 205: 867-75.

18. Qin Y, Yuan M, Li L, et al. Use of polylactic acid/polytrimethylene carbonate blends membrane to prevent postoperative adhesions. J Biomed Mater Res Part B Appl Biomater 2006; 79B: 312-9.

19. Tyson T, Finne-Wistrand A, Albertsson AC. Degradable porous scaffolds from various L-lactide and trimethylene carbonate copolymers obtained by a simple and effective method. Biomacromolecules 2009; 10: 149-54.

20. Zhang Z, Kuijer R, Bulstra SK, Grijpma DW, Feijen J. The in vivo and in vitro degradation behavior of poly(trimethylene carbonate). Biomaterials 2006; 27: 1741-8.

21. Wach RA, Adamus A, Olejnik AK, Dierzawska J, Rosiak JM. Nerve guidance channels based on PLLA-PTMC biomaterial. J Appl Polym Sci 2013; 127: 2259-68.

22. Battiston B, Geuna S, Ferrero M, Tos P. Nerve repair by means of tubulization: literature review and personal clinical experience comparing biological and synthetic conduits for sensory nerve repair. Microsurgery 2005; 25 : 258-67.

23. Duda A, Penczek S. Kinetics of polymerization with reversible deactivation due to aggregation of active centers: analytical vs. numerical solution for the l-caprolactone/dialkylaluminium alkoxide system. Macromol Chem Commun 1994; 15: 559-66.

24. Kowalska-Ludwicka K, Grobelski B, Cala J, Sygut D, Jesionek-Kupnicka D, Kolodziejczyk M. Modified bacterial cellulose tubes for regeneration of damaged peripheral nerves. Arch Med Sci in press.

25. Rowshan K, Jones NF, Gupta R. Current surgical techniques of peripheral nerve repair. Oper Tech Orthop 2004; 14: 163-70.

26. Medical Research Council, Nerve Injuries Committee. Aids to Investigation of Peripheral Nerve Injuries. MRC War memorandum No. 7 His Majesty's Stationery Office, 1943. London, Balliere Tindall, 1986.

27. Pego AP, Poot AA, Grijpma DW, Feijen J. Biodegradable elastomeric scaffolds for soft tissue engineering. J Control Release 2003; 87: 69-79.

28. Plikk P, Malberg S, Albertsson AC. Design of resorbable porous tubular copolyester scaffolds for use in nerve regeneration. Biomacromolecules 2009; 10: 1259-64.

29. Jozwiakowska J, Wach RA, Rokita B, et al. Influence of electron beam irradiation on physicochemical properties of poly(trimethylene carbonate). Polym Degrad Stab 2011; 96: 1430-7.

30. Nugroho P, Mitomo H, Yoshii F, Kume T. Degradation of poly(L-lactic acid) by gamma-irradiation. Polym Degrad Stab 2001; 72: 337-43.

31. Adamus A, Wach RA, Olejnik AK, Dzierzawska J, Rosiak JM. Degradation of nerve guidance channels based on a poly(L-lactic acid) poly(trimethylene carbonate) biomaterial. Polym Degrad Stab 2012; 97: 532-40.

32. Kryger GS, Kryger Z, Zhang F, Shelton DL, Lineaweaver WC, Buncke HJ. Nerve growth factor inhibition prevents traumatic neuroma formation in the rat. J Hand Surg 2001; 26: 635-43.

33. Ro LS, Chen ST, Tang LM, Jacobs JM. Effect of NGF and anti-NGF on neuropathic pain in rats following chronic constriction injury of the sciatic nerve. Pain 1999; 79: 265-74.

34. Fu K, Pack DW, Klibanov AM, Langer R. Visual evidence of acidic environment within degrading poly(lactic-co-glycolic acid) (PLGA) microspheres. Pharm Res 2000; 17: 100-6.
35. Pego AP, Poot AA, Grijpma DW, Feijen J. In vitro degradation of trimethylene carbonate based (co)polymers. Macromol Biosci 2002; 2: 411-9. 